\title{
Visual Controllers for Relative Positioning in Indoor Settings
}

\author{
Luis Mejias $^{1}$ and Pascual Campoy ${ }^{2}$
}

\begin{abstract}
This paper presents an approach to visually control a quadrotor relative to known landmarks. Relative positioning is achieved here by a new decoupled approach to image based visual servoing (IBVS), in which rotational and translational coupling is minimised by introducing a decay function that is proportional to feature location in the image. Simulations and experiments were conducted to validate the proposed method. Results show that our method has benefits over standard decoupled IBVS by ensuring smoother trajectories in the image plane as well as in task space, minimising the risk of features leaving the image plane.
\end{abstract}

\section{INTRODUCTION}

Controlling the position of a robot relative to known objects or landmarks is one possible approach to move and localise such a robot in environments which lack of absolute positioning [1]. The term visual navigation is often used to denote the use of cameras for this purpose. The accuracy and robustness of the visual controller used for this type of navigation is critical to ensure drift-free long-term navigation. This type of landmark-based relative navigation can be combined with well established SLAM methods to achieve better global localisation. SLAM approaches are not immune to drift and can rapidly be affected in this context due to the unconstrained 3D motion of a camera attached to a drone. Assuming, drift can be minimised but not removed completely from the odometry estimation, an approach in which ground landmarks are used to correct the drift (through relative positioning) will lead to minimal deviation from the desired trajectory. Our proposed visual controller involves an intuitive approach that models the way human pilots navigate. Human pilots flying under Visual Flight Rules (VFR) observe their surroundings and compare landmarks (both natural and artificial) to the landmarks printed on maps along their pre-planned route.

In this context, detection and relative positioning with regard to landmarks poses some challenges since the process is performed while the vehicle is in motion. This motion will impact the initial conditions of the controller (often linear) used for the relative positioning. Linearisation of the dynamics for control purposes is often necessary (although not always desirable) in order to design practical controllers to deal with highly non-linear systems such as mutltirotors. For example, many applications of visual servoing or visual

\footnotetext{
1 is with the School of Electrical Engineering and Computer Science, Queensland University of Technology, Brisbane, QLD 4000, Australia luis.mejiasequt.edu.au

2 is with the Computer Vision and Aerial Robotics (CVAR) Group, Centre for Automation and Robotics (UPM-CSIC), Calle José Gutiérrez Abascal 2, Madrid (Spain). pascual. campoy@upm.es
}

navigation such as object following [2], [3], [4], object grasping and manipulation [5], [6], landing [7], and inspection tasks [8], [9], [10] among others, rely on linearisation of the dynamics around an operating point (i.e hover or near hover).

Operating at flight envelops away from the operating point, poses challenges in terms of control, stability and robustness. A moving vehicle navigating in unknown environments is an example of this case. Therefore, in this paper we investigate a technique that allows a quadrotor to move at velocities around $0.6 \mathrm{~m} / \mathrm{s}$ and perform a visual servoing task to localise itself relative to known landmarks. Smoother trajectories and faster converge leads to better relative localisation which in turn improve the navigation task by correcting apparent drift. In this paper, we focus on the core technique (visual servoing) that would allow better visual relative positioning w.r.t to landmarks. We don't explicitly address the visual navigation or SLAM problem. The idea behind our approach is to separate vehicle translation and rotation by introducing a function that depends of feature location in the image. In simple terms, the quadrotor will not rotate until translational motion brings the features close to their desired location in the image. Implementing this approach contributes the following benefits:

- Feature motion tend towards linear trajectories in the image over a wide range of initial conditions.

- Minimise risk of feature loss (out of FOV), ensuring feature visibility at all times.

- Smoother and more continuous trajectories in task space which leads to smoother flights.

This paper is organised as follows: Section II provides a brief overview of the related work, Section III presents a brief dynamic model and coordinate conventions used in this paper. Section IV introduces the IBVS approach and Section $\mathrm{V}$ details our proposed approach. Simulations and flight tests are presented in Section VI. Section VII concludes the paper and presents future research directions.

Robust relative positioning using landmarks provides an immediate drift correcting effect when visual odometry or SLAM are used for long-term navigation

\section{RELATED WORK}

Traditionally landmark-based navigation has been approached in two ways, i) as a simultaneously mapping and localisation problem, ii) as the process of constructing some type of topological or metric-based maps [11]. However, there exist methods that do not explicitly reconstruct the scene or create a map. For instance, in [12], [13] a vehicle was able to guide itself to a home position by matching bearings extracted from a sequence of panoramic images 
with the local vehicle state. The vehicle did not need to be aware of its position and orientation and did not need to reconstruct the scene. Zhu and Deng [14] presented an approach in which detected landmarks were used to estimate aircraft local position and then correct the flight trajectory, however, GPS was still used as the primary navigation sensor. These methods make use of a control law that minimises the error between pre-learned states extracted from images to the current vehicle state. Closer to the approach presented here, classic visual servoing was used to navigate mobile ground robots in [15], [16]. Their approach was the application of the servoing technique itself without consideration of the rotation and translation coupling. To the best of our knowledge, no other work has tried to further enhance decoupled visual servoing approaches, and in particular, not in the aerial domain. Key research works in aerial visual servoing have approached the problem assuming hover or slow translational motion [17], [18], [19]. They have developed important insights into the theory and stability of such approach. In this paper, we target scenarios in which the initial conditions involve arbitrary speeds and relative orientations between target and UAV.

\section{Vehicle Dynamic Model}

The vehicle position w.r.t to the navigation frame $N=$ $\left(n_{1}, n_{2}, n_{3}\right)$ is denoted by the vector $d_{n}^{c}=\left(n_{1}^{\prime}, n_{2}^{\prime}, n_{3}^{\prime}\right)$. This vector is usually provided by an absolute sensor such as GPS or a dead-reckoning approach such as visual odometry. The camera frame and body-fixed frame are colocated and denoted by $\mathbb{C}$. The target coordinate system is denoted by $\Gamma$ and the target position relative to the vehicle is denoted by the vector $d_{\Gamma}^{c}=\left(\tau_{1}^{\prime}, \tau_{2}^{\prime}, \tau_{3}^{\prime}\right)$. The position of the target in the navigation frame is given by the vector $d_{n}^{\Gamma}$ which involves a rotation (R) and translation (T) from $\Gamma$ to $N$, such that $R, T: \Gamma \rightarrow N$. Camera velocities $\nu=(v, \omega)$ are $v=\left(v_{x}, v_{y}, v_{z}\right)$ (linear) and $\omega=\left(\omega_{x}, \omega_{y}, \omega_{z}\right)$ (angular). Orientation of the camera is given by Euler angles roll, pitch and yaw $(\phi, \theta, \psi)$, respectively. Body-fixed velocity of the vehicle is denoted by $v^{c}$ and inertial velocity by $v^{I}=\dot{d}_{n}^{c}$. Intuitively, we can see that by knowing the position $\left(d_{n}^{\Gamma}\right)$ of the target or landmark in the $N$ frame we can solve for $d_{n}^{c}$, which is the position of the vehicle that will likely be affected by drift or sensor drop-outs. The multirotor dynamics are expressed using standard rigid body dynamics

$$
\begin{aligned}
\dot{d}_{n}^{c} & =R v^{c} \\
\dot{R} & =R[\omega]_{\times} \\
m \dot{v}^{c} & =-m[\omega]_{\times} v^{c}+F \\
F & =-T e_{3}+m g R^{T} e_{3} \\
I \dot{\omega} & =-[\omega]_{\times} I \omega+\tau
\end{aligned}
$$

where $e_{3}=(0,0,1)$ is a unit vector in $\mathbb{R}^{3}, g$ is the gravity, $m$ is vehicle mass, $I$ is the inertia matrix $\in \mathbb{R}^{3},[w]_{\times}$denotes the skew symmetric matrix such that $[w]_{\times} v=w \times v, T \in$ $\mathbb{R}$ is the magnitude of the total thrust in the direction $e_{3}$ and $\tau \in \mathbb{R}^{3}$ (no subscript) are the force and torque vectors

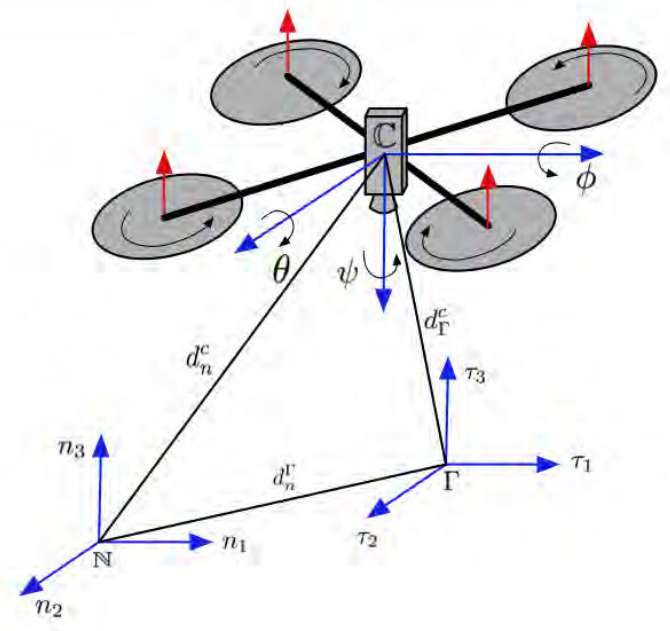

Fig. 1. Coordinate frame definitions. Vehicle body frame and camera frame (C) are co-located, therefore for simplicity we express linear and angular states and variables associated with the vehicle in the camera frame.

expressed in body frame. Control is achieved by changing torque control $\tau \in \mathbb{R}^{3}$ to align the force (or total thrust vector) $F$ in order to track the goal trajectory.

\section{Visual CONTROLleRs}

\section{A. Camera model}

We follow a common representation of the camera geometry (central perspective imaging model), modelling the imaging sensor as a pinhole camera [20], [21]. Using this model we map points from a 3-dimensional space to a 2dimensional image plane, and vice versa. Thus, 3D points (and their motion) can be mapped into 2D points (image features) in the image plane. This mapping allow us to relate camera velocity to feature velocity (commonly in normalised image coordinates) using the following relationship $\dot{s}=L_{s} \nu$, where $s=(\bar{u}, \bar{v})$ are the cartesian pixel positions of feature points, $L_{s}$, often referred as image Jacobian or interaction matrix, is a matrix that is function of s, camera focal length and the relative distance between $3 \mathrm{D}$ points and camera, and finally $\nu=\left(v_{x}, v_{y}, v_{z}, \omega_{x}, \omega_{y}, \omega_{z}\right) \in \mathbb{R}^{6}$ is the vector that encodes the camera's linear and angular velocities. There are mainly two forms of visual control in the literature. They differ in the way the observed image features are used to generate the control signal. Position-based visual servoing (PBVS) uses image features to estimate target pose (in 3D) and then perform the control task in SE(3) (task space). Image-based visual servoing (IBVS) uses image features directly (no relative pose is estimated) to then perform the control task in the image plane space $\mathrm{SE}(2)$. For details on these definitions and their associated mathematical derivation refer to [20], [21], [22]

\section{B. IBVS}

Classic IBVS techniques use image features that are 2D projections of lines, circles or 3D objects to create a control law that brings image features to their desired location in the 
image. An object can be represented in the image plane by a set of features $s_{i}=\left(\bar{u}_{i}, \bar{v}_{i}\right), i=1 . . n$. Similarly the desired position of the object in the image plane can be represented by a set of desired features $s_{i}^{*}=\left(\bar{u}_{i}^{*}, \bar{v}_{i}^{*}\right), i=1 . . n$. Thus, the control objective is to position the camera such that the elements of $s_{i}$ coincide or match element-wise $s_{i}^{*}$ using an error signal such as $e_{i}=s_{i}-s_{i}^{*}$. In general terms, the classical definition of IBVS consist of

$$
\nu=-\lambda \hat{L}^{+} e
$$

where $\hat{L}_{s}^{+}$is an approximation of the pseudo-inverse of $L_{s}, \lambda$ is a positive gain and $\nu$ the velocity or control vector. An approximation for $L_{s}$ is used because visual servoing approaches require 3D parameters that are not directly measurable from the image. This controller will generate linear and angular velocities such that the image features move toward the desired position in the image. However, there exist the possibility that no camera motion will result in achieving the required image feature motion. In this case, a sub-optimal solution will be found that minimises the norm of the feature velocity error.

\section{Advanced Approaches}

Advances approaches have been proposed in the literature to combine the advantages of PBVS and IBVS while avoiding their shortcomings. For instance, IBVS is a challenging control problem since the image features are a highly nonlinear function of camera pose, while PVBS requires accurate calibration parameters and estimation of $3 \mathrm{D}$ information. There has been numerous efforts to find a set of optimal features that ensure robust camera motions by combining and/or decoupling some degrees of freedom. Hybrid visual servoing approaches are an example of these techniques.

For instance, they partition the interaction matrix such that

$$
\begin{aligned}
\dot{s} & =L_{s} \nu \\
& =\left[\begin{array}{ll}
L_{v} & L_{\omega}
\end{array}\right]\left[\begin{array}{c}
v \\
\omega
\end{array}\right] \\
& =L_{v} v+L_{\omega} \omega
\end{aligned}
$$

where $L_{v}$ and $L_{\omega}$ are the translational and rotational components of the interaction matrix, respectively. The control law $\left(\nu=-\lambda \hat{L}^{+} e\right)$, then becomes

$$
v=-\hat{L}_{v}^{+}\left(\lambda e+L_{\omega} \omega\right)
$$

where $v$ involves only the translational velocities. This equation combines the original error with the error that would be induced by the rotational motion due to $\omega$. A common choice for $\omega$ is $\omega=-\lambda \theta u$, where $\theta u$ is a error term obtained either in 3D or 2D (image plane). Hybrid approaches aim at decoupling the rotational motions from the translational ones by combing features in 2-D, and in part in 3-D. The goal is to find features that relate to one degree of freedom to convert the problem to a direct, and simple linear control problem [23], [24]. This property is of particular interest for a highly non-linear dynamic systems such as a quadrotor. Previous works have used this decoupled approach to control



Fig. 2. Camera trajectory when both controllers are simulated under similar initial conditions $\left(\phi_{1}=0.26, \phi_{2}=1.3\right)$. $\phi$ is the angle between camera and target (in rad). It can be seen how our proposed controller (16) can deal with large $\phi$ values. Hybrid controller with $\phi_{2}$ will cause features to leave the camera FOV.

quadrotors around an operating point (specific flight state such as hover) [19], [25]. By decoupling linear motion (linear velocity in $x, y, z$ ) and rotational motion (angular velocity in $z$ ), the following control law has provided acceptable results in near hover conditions [19], [25]

$$
\begin{aligned}
v_{x y} & =-\hat{L}_{x y}^{+}\left(\lambda e+L_{z}\left[v_{z} \omega_{z}\right]^{T}\right) \\
\omega_{z} & =\lambda_{1}\left(\psi_{t}^{*}-\psi_{t}\right) \\
v_{z} & =\lambda_{2} \ln \frac{\sigma^{*}}{\sigma}
\end{aligned}
$$

where $L_{x y}$ and $L_{z}$ are formed by extracting specific columns from $L_{s}$. (see [22] for details), $\sigma$ is the area of the shape enclosed by a set of features $s_{i}=\left(\bar{u}_{i}, \bar{v}_{i}\right), \sigma^{*}$ its desired value, $\psi_{t}$ is the orientation of the shape (or target) enclosed by the same set of features, $\psi_{t}^{*}$ its desired value and $\lambda_{1}, \lambda_{2}$ are positive gains. As previously mentioned (10), $v=v_{x y}$ will still have rotational components due to the coupling with $\omega_{z}$, that in hover (slow translation and rotation) can still produce stable behaviour due to the small angle assumption [25], but unfortunately these conditions are not targeted in this paper. The issue arise when the quadrotor is (i) flying at an arbitrary height and the ratio $\frac{\sigma^{*}}{\sigma} \gg 1$ or $1 \ll \frac{\sigma^{*}}{\sigma}$ or simply the difference in height is significant, (ii) when $\left|\left(\psi_{t}^{*}-\psi_{t}\right)\right| \gg$ 0 , i.e, difference in orientation between quadrotor and target is significant. To illustrate this using the toolbox in [20], we simulated (11) using a free flying camera with two different initial conditions for initial heading between camera and target $\left(\phi_{1}, \phi_{2}\right)$.

Fig. 2 and 3 show the trajectory of the camera in task space and features in the image plane, respectively. The hybrid approach with $\phi_{2}$ moves away from the starting position, then converges to the final position, but the features leave the image (Fig. 3). Our proposed approach can compensate for this situation ensuring observability and continuous control. 
To avoid undesired trajectories as result of the quadrotor detecting a target with arbitrary orientation and height, our approach compensates for the rotational components that cause undesirable non-linear trajectories, that may also cause the features to leave the camera field of view (Fig. 3).

\section{Proposed ApproACH}

The idea behind our approach is to introduce a term in (11) that is function of the feature location so that the rotational components are minimised until the features are close enough to their desired location. Our function has the form

$$
F=\left\{\begin{array}{l}
\lim _{s \rightarrow s *} f(s)=1 \\
\lim _{s \rightarrow 1} f(s)=0
\end{array}\right.
$$

where $s$ is the feature location in normalised image coordinates ( $s \rightarrow 1$ mean features moving toward the edges) and $s^{*}$ their desired values. As the features move towards their desired values the function $f(s)$ will tend to one allowing rotation to occur. Conversely, only translation will be allowed if features move away from their desired values. Exponential or decay functions have this property in which the growth or decay rate can be set, in our case, proportional to the error $e=s-s^{*}$ such that $f(s)=k_{1} e^{k_{2}(1-e)}$ where $k_{1}$ and $k_{2}$ are gains that regulate rate of decay and upper/lower function limits. The following step is to introduce this function in (11) such that

$$
\begin{aligned}
\bar{v} & =f(s)\left[v_{z} \omega_{z}\right]^{T} \\
v_{x y} & =-\hat{L}_{x y}^{+}\left(\lambda e+L_{z} \bar{v}\right)
\end{aligned}
$$

by introducing this new function, the component $L_{z} \bar{v}$ of (16) has little effect on translation until the features are close enough to their desired values. It has also the effect of compensating for significant altitude differences in initial

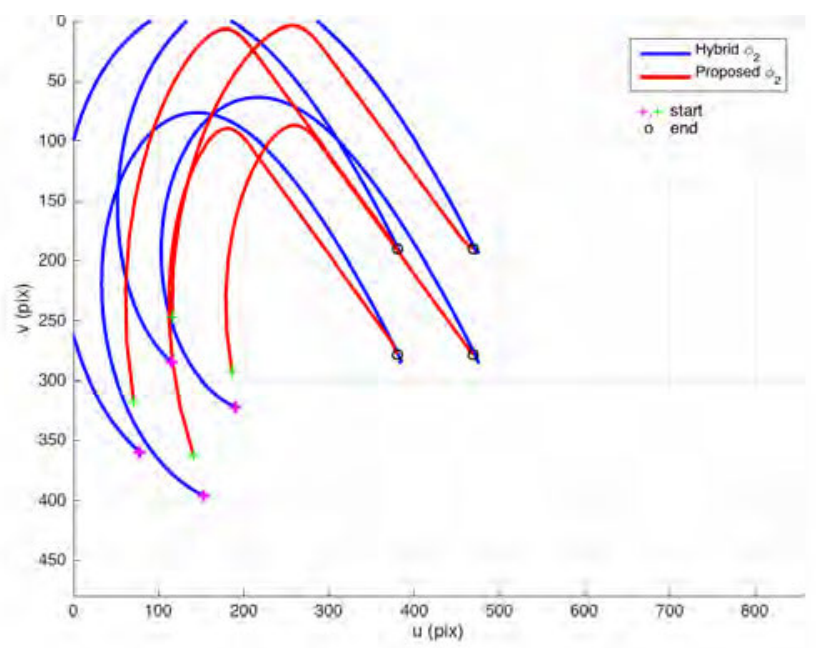

Fig. 3. Feature trajectories in the image for angle $\phi_{2}$. Using trajectories 2 and 4 from Fig. 2. It can be seen how our proposed controller (16) can deal with large $\phi$ values and prevent features from leaving the image plane. Starting position denoted by + and final by $\circ$. conditions. This work shows the feasibility of introducing this new approach for controlling a quadrotor, an extension of this work will include different forms of $f(s)$ that include the altitude component as well as a proof of stability. Simulation of this new behaviour is shown in Fig. 3. It is important to note the following observations gathered during controller design and simulation:

- Small heading differences $(\phi)$ will have no effect on performance of both controllers. A small difference is representative of near hover and target aligned with quadrotor.

- For large $(\phi)$, our controller managed to keep features in the field of view, while the Hybrid decoupled controller cannot.

- On both cases, tuning the gains is an extremely onerous process. Good performance can be achieve for a particular condition, but there is no guaranteed performance for arbitrary $(\phi)$ and altitude.

- Alternative gain tuning methods could be quite beneficial for these type of controllers.

\section{Vi. Simulation and Flight Test}

The control architecture follows a standard IBVS scheme in which images are processed onboard in a separate computer, then control signals are sent via dedicated communication channel to the autopilot. This architecture is shown in Fig. 4. The main advantage of using such a configuration is the flexibility and transparency between simulation and flight test. By using ROS (Robot Operating System) we were able to send same ROS message between the simulated quad and the real quad, reducing the overhead during development and testing.

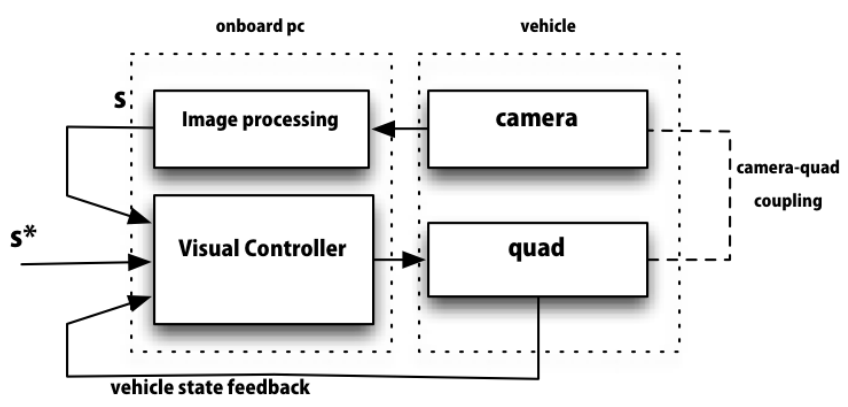

Fig. 4. System architecture. The same architecture was used for simulations and flight tests. Onboard pc runs ROS (Robot Operating System)

The controller was implemented using a combination of developed routines combined with the package VISP [26]. The image processing was performed using a ROS wrapper to detect Aruco markers [27]. Similarly, by using standard ROS messages we were able to transparently process synthetic images and real ones generating the same references for the controller. Simplifying the detection front-end by using Aruco markers allow us to assess independently the performance of the controller. We acknowledge in many 

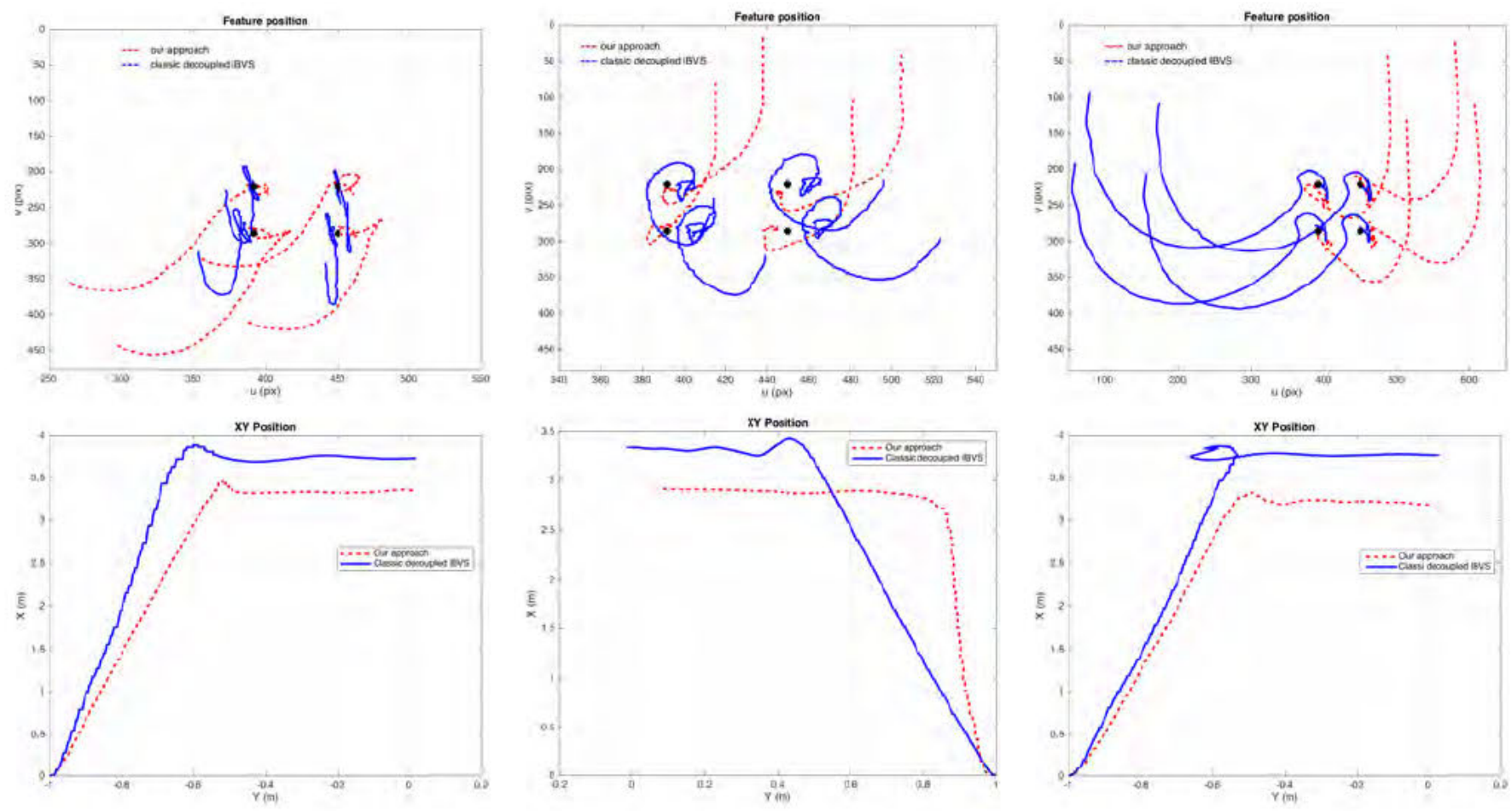

Fig. 5. Feature position in image plane and XY quadrotor position in work space. Predominantly linear trajectories in the image are observed using our approach, corresponding to less overshot and smoother quadrotor trajectories in task space. Our approach (--) and classic decoupled IBVS (-) are shown.

practical scenarios feature detection and tracking might be challenging, however there exist a myriad of excellent feature detectors proven to work in practice [28].

Prior to flight testing, we conducted a series of simulations using ROS, Gazebo and the open source Hector quadrotor model detailed here [29]. Using synthetic images from an onboard downward facing camera we were able run the Aruco detector and use our visual controller to test the validity of our approach. The transition between simulation and flight testing was straight forward only requiring the execution of an alternative ROS node to communicate either with the quadrotor using the bebop_autonomy [30] package or the Hector package.

\section{A. Experimental platform}

Our experimental platform is the Parrot BeBop2 equiped with a Parrot S.L.A.M.DUNK navigation onboard computer. This computer features a NVIDIA Tegra K1 mobile processor and runs ROS Indigo. The communication between onboard computer and BeBop2 is done via USB. Using the package bebop_autonomy [30] full control and access to the Bebop2 states can be achieved. The interface with external ROS packages or nodes is straightforward through the bebop_autonomy ROS package. The camera was set downward looking with a $856 \times 480$ resolution transmitting full color video. Aruco detector [27] and servoing algorithms were executed on the S.L.A.M.DUNK which was remotely accessed via ssh. The combination Bebop2, S.L.A.M.DUNK and ROS reduced significantly the engineering and development time associated with experimentation.

\section{B. Flight Test}

We designed a series of flying scenarios to test the proposed approach. Four circuits were designed, one for controller design and validation and three for practical demonstration. Here, we present the results (Figs. $5 \& 6$ ) from the validation scenario depicted in Fig. 7. In this scenario, the quad will start flying diagonally towards the marker, once detected the visual servoing task will move the target to the centre of the image, positioning the quad on top of the marker. The average quad longitudinal velocity was $0.6 \mathrm{~m} / \mathrm{s}$. We conducted 30 flights with the quadrotor starting either right (15) or left (15) the centre line joining target and workspace origin. No external tracking system (Vicon or Optitrack) was used in these experiments. Therefore, the quad states depicted in our plots are purely from onboard odometry. These values are used for reference, and to show the location of the quad relative to the target.

The main advantage of our approach can be observed in Fig. 5. Both, in the image plane and in task space, trajectories tend to be more linear and smoother. It can be seen that features tend to leave the camera fov, if the classic hybrid decoupled IBVS is used (top-right Fig. 5), whereas our approach minimises this risk through translation followed by rotation based on feature location. The resultant $\mathrm{XY}$ trajectories for a set of experiments are shown in Fig 6 . It can be observed that trajectories on the right plot exhibit consistent behaviour with less overshot compared with left plot. Video footage from additional flight tests can be accessed at https://youtu.be/ajEN90CSc08 

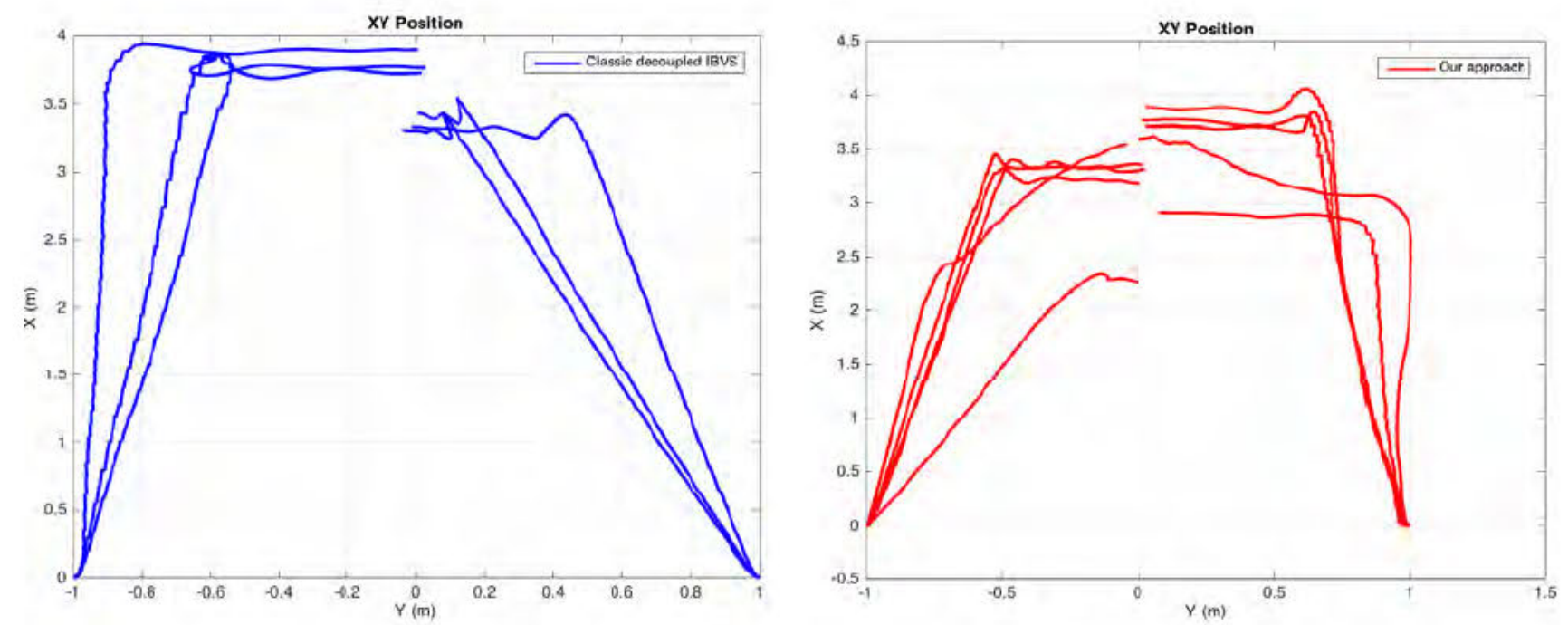

Fig. 6. Example of two sets of flight tests. Figure on the left correspond to the classic decoupled IBVS using (11) and figure on the right our proposed approach (16).

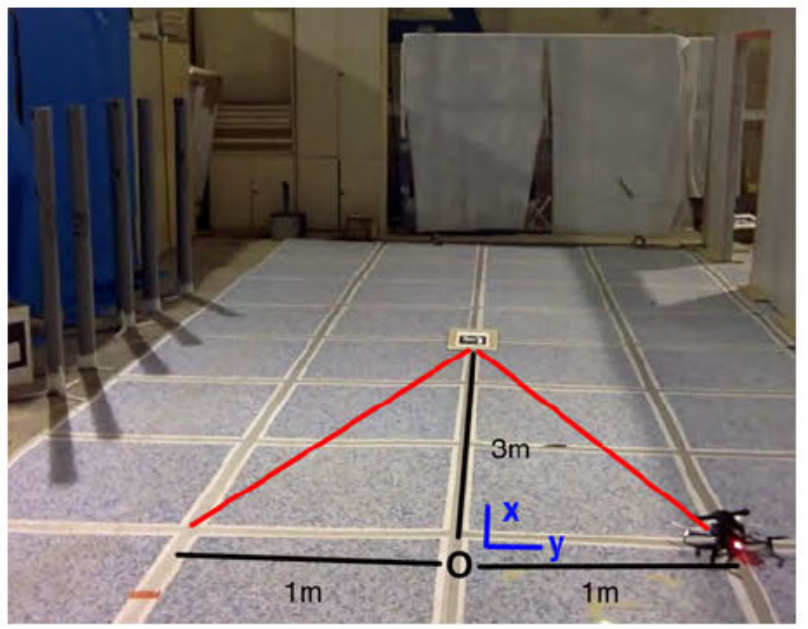

Fig. 7. Actual picture of the workspace showing the dimensions between target and quad. This setup was used to validate, design and tune our visual controller. Initial quad position can be either $1 \mathrm{~m}$ right or $1 \mathrm{~m}$ left from the centre line and $3 \mathrm{~m}$ behind the target, that is, target at $(3,0)$, and quad $(0,1)$ or $(0,-1)$.

\section{CONCLUSIONS}

In this paper, we proposed a new exponentially decoupled Image-Based Visual Servoing approach for landmark-based navigation. The method was extensively compared with the classic decoupled IBVS, showing more linear and smoother trajectories in both task and feature space (Fig. 5). One of the key properties of the proposed approach is the ability to work in conditions other than hover or low speeds, and still providing more desirable trajectories ensuring feature visibility. Future work includes investigations into stability, defining visibility contours in the image and adding altitude components in the decay function $f(s)$.

\section{ACKNOWLEDGMENT}

This work was supported in part by an Australian Endeavour Executive Fellowship and the Queensland University of Technology professional development program (PDL). We would also like to acknowledge the support from University of Toronto Institute for Aerospace Studies and the Computer Vision Group at Universidad Politécnica de Madrid.

\section{REFERENCES}

[1] L. Matthies, "Dynamic stereo vision," CMU-CS-89-195, Carnegie Mellon University. Computer Science Department, 1989.

[2] J. Pestana, J. L. Sanchez-Lopez, P. Campoy, and S. Saripalli, "Vision based gps-denied object tracking and following for unmanned aerial vehicles," in Safety, security, and rescue robotics (ssrr), 2013 ieee international symposium on. IEEE, 2013, pp. 1-6.

[3] J. Thomas, J. Welde, G. Loianno, K. Daniilidis, and V. Kumar, "Autonomous flight for detection, localization, and tracking of moving targets with a small quadrotor," IEEE Robotics and Automation Letters, vol. 2, no. 3, pp. 1762-1769, 2017.

[4] S. Li, T. Liu, C. Zhang, D.-Y. Yeung, and S. Shen, "Learning unmanned aerial vehicle control for autonomous target following," arXiv preprint arXiv:1709.08233, 2017.

[5] J. Thomas, G. Loianno, K. Sreenath, and V. Kumar, "Toward image based visual servoing for aerial grasping and perching," in Robotics and Automation (ICRA), 2014 IEEE International Conference on. IEEE, 2014, pp. 2113-2118.

[6] V. Lippiello, J. Cacace, A. Santamaria-Navarro, J. Andrade-Cetto, M. A. Trujillo, Y. R. Esteves, and A. Viguria, "Hybrid visual servoing with hierarchical task composition for aerial manipulation," IEEE Robotics and Automation Letters, vol. 1, no. 1, pp. 259-266, 2016.

[7] D. Lee, T. Ryan, and H. J. Kim, "Autonomous landing of a vtol uav on a moving platform using image-based visual servoing," in Robotics and Automation (ICRA), 2012 IEEE International Conference on. IEEE, 2012, pp. 971-976.

[8] N. Metni and T. Hamel, "A uav for bridge inspection: Visual servoing control law with orientation limits," Automation in construction, vol. 17, no. 1, pp. 3-10, 2007.

[9] I. Sa, S. Hrabar, and P. Corke, "Inspection of pole-like structures using a vision-controlled vtol uav and shared autonomy," in Intelligent Robots and Systems (IROS 2014), 2014 IEEE/RSJ International Conference on. IEEE, 2014, pp. 4819-4826. 
[10] O. Araar and N. Aouf, "Visual servoing of a quadrotor uav for autonomous power lines inspection," in Control and Automation (MED), 2014 22nd Mediterranean Conference of. IEEE, 2014, pp. 14181424.

[11] D. Fassbender, M. Kusenbach, and H. J. Wuensche, "Landmark-based navigation in large-scale outdoor environments," in 2015 IEEE/RSJ International Conference on Intelligent Robots and Systems (IROS), Sept 2015, pp. 4445-4450.

[12] A. A. Argyros, K. E. Bekris, and S. C. Orphanoudakis, "Robot homing based on corner tracking in a sequence of panoramic images," in Proceedings of the 2001 IEEE Computer Society Conference on Computer Vision and Pattern Recognition. CVPR 2001, vol. 2, 2001, pp. II-3-II-10 vol.2.

[13] B. KE., A. A., and K. LE., "Exploiting panoramic vision for anglebased robot homing," in Lecture Notes in Computer Science. Springer, 2006, vol. 33, pp. 229-251.

[14] H. Zhu and L. Deng, "A landmark-based navigation method for autonomous aircraft," Optik - International Journal for Light and Electron Optics, vol. 127, no. 7, pp. 3572 - 3575, 2016. [Online]. Available: http://www.sciencedirect.com/science/article/pii/S0030402616000437

[15] A. Begum, M. Lee, and Y. J. Kim, "A simple visual servoing and navigation algorithm for an omnidirectional robot," in 2010 3rd International Conference on Human-Centric Computing, Aug 2010, pp. $1-5$.

[16] G. L. Mariottini and D. Prattichizzo, "Image-based visual servoing with central catadioptric cameras," International Journal of Robotics Research, vol. 27, no. 1, pp. 41-56, 2008.

[17] T. Hamel and R. Mahony, "Visual servoing of an under-actuated dynamic rigid-body system: an image-based approach," Robotics and Automation, IEEE Transactions on, vol. 18, no. 2, pp. 187 -198, Apr. 2002.

[18] E. Altuğ, "Vision based control of unmanned aerial vehicles with applications to an autonomous four rotor helicopter," Ph.D. dissertation, Department of Mechanical Engineering and Applied Mechanics, University of Pennsylvania, December 2003.

[19] R. Mahony and T. Hamel, "Image-based visual servo control of aerial robotic systems using linear image features," IEEE Transactions on Robotics, vol. 21, no. 2, pp. 227-239, April 2005.

[20] P. Corke, Robotics, Vision and Control Fundamental Algorithms in MATLAB, ser. Springer Tracts in Advanced Robotics, Vol. 73. Springer-Verlag Berlin Heidelberg, 2011.

[21] F. Chaumette and S. Hutchinson, "Visual servo control. i. basic approaches," Robotics Automation Magazine, IEEE, vol. 13, no. 4, pp. $82-90$, dec. 2006.

[22] - "Visual servo control. ii. advanced approaches [tutorial]," Robotics Automation Magazine, IEEE, vol. 14, no. 1, pp. 109 -118, march 2007.

[23] E. Malis, F. Chaumette, and S. Boudet, "2-1/2D visual servoing," IEEE Transactions on Robotics and Automation, vol. 15, no. 2, pp. 238-250, April 1999.

[24] P. Corke and S. Hutchinson, "A new partitioned approach to imagebased visual servo control," Robotics and Automation, IEEE Transactions on, vol. 17, no. 4, pp. $507-515$, Aug. 2001.

[25] O. Bourquardez, R. Mahony, N. Guenard, F. Chaumette, T. Hamel, and L. Eck, "Image-based visual servo control of the translation kinematics of a quadrotor aerial vehicle," IEEE Transactions on Robotics, vol. 25, no. 3, pp. 743-749, June 2009.

[26] E. Marchand, F. Spindler, and F. Chaumette, "Visp for visual servoing: a generic software platform with a wide class of robot control skills," IEEE Robotics and Automation Magazine, Special Issue on "Software Packages for Vision-Based Control of Motion”, vol. 12, no. 4, pp. 40-52, December 2005.

[27] S. Garrido-Jurado, R. Muñoz-Salinas, F. Madrid-Cuevas, and M. Marín-Jiménez, "Automatic generation and detection of highly reliable fiducial markers under occlusion," Pattern Recognition, vol. 47, no. 6, pp. 2280 - 2292, 2014. [Online]. Available: http://www.sciencedirect.com/science/article/pii/S0031320314000235

[28] C. Kanellakis and G. Nikolakopoulos, "Survey on computer vision for uavs: Current developments and trends," Journal of Intelligent \& Robotic Systems, vol. 87, no. 1, pp. 141-168, Jul 2017. [Online]. Available: https://doi.org/10.1007/s10846-017-0483-z

[29] J. Meyer, A. Sendobry, S. Kohlbrecher, U. Klingauf, and O. von Stryk, "Comprehensive simulation of quadrotor uavs using ros and gazebo," in Simulation, Modeling, and Programming for Autonomous
Robots, I. Noda, N. Ando, D. Brugali, and J. J. Kuffner, Eds. Berlin, Heidelberg: Springer Berlin Heidelberg, 2012, pp. 400-411.

[30] M. Monajjemi, "bebop_autonomy," http://bebopautonomy.readthedocs.io/en/latest/index.html, Feb. 2018. 